\title{
Green fluorescent protein as a reporter for the spatial and temporal expression of actIII in Streptomyces coelicolor
}

\author{
Fernando Santos-Beneit ${ }^{1}$ (D) Jeff Errington ${ }^{1}$
}

Received: 13 February 2017 / Revised: 1 March 2017 / Accepted: 7 March 2017 / Published online: 22 March 2017

(C) The Author(s) 2017. This article is an open access publication

\begin{abstract}
Polyketides constitute a large group of structurally diverse natural products with useful biological activities. Insights into their biosynthetic mechanisms are crucial for developing new structures. One of the most studied model polyketide is the blue-pigmented antibiotic actinorhodin, produced by Streptomyces coelicolor. This aromatic polyketide is synthesized by minimal type II polyketide synthases and tailoring enzymes. The ActIII actinorhodin ketoreductase is a key tailoring enzyme in actinorhodin biosynthesis. Previous papers have reported contradictory findings for localization of the protein in the cytoplasmic fraction or associated with the cell wall. We have now used green fluorescent protein as a reporter to analyse the spatial and temporal expression of actIII (SCO5086) in S. coelicolor under actinorhodin producing and non-producing conditions. We provide evidence in support of ActIII being a cytosolic protein, with limited if any association with the membrane or cell wall.
\end{abstract}

Keywords Polyketides - Actinorhodin - Streptomyces coelicolor $\cdot$ ActIII $\cdot$ ActA $\cdot$ SCO5086 $\cdot$ SCO5083

\section{Introduction}

Polyketides are an important class of natural products with many different biological functions that can be produced by

Communicated by Yusuf Akhter.

Fernando Santos-Beneit

fernando.santos-beneit@ncl.ac.uk

Centre for Bacterial Cell Biology, Institute for Cell and Molecular Biosciences, Medical School, Newcastle University, Newcastle upon Tyne, UK bacteria, fungi and plants (Hopwood 1997). The enzymes that synthesize these complex molecules are known as polyketide synthases. These proteins are analogous to the closely related fatty acid synthases which use the decarboxylation of $\alpha$-carboxylated precursors to drive the synthesis of carbon chains (Keatinge-Clay et al. 2004). Actinorhodin provides a classic example of how these secondary metabolites are synthesized and regulated. This polyketide antibiotic is produced by the soil bacterium Streptomyces coelicolor and, as a blue isochromanequinone compound, provides a convenient model for studies of regulation (Rawlings 1999). The actinorhodin biosynthetic gene cluster (Act) has been extensively studied and the function of most genes characterized (Malpartida and Hopwood 1986; FernandezMoreno et al. 1994). The carbon backbone of actinorhodin is assembled through the cooperation of a ketosynthase/ chain length factor (KS-CLF; actI-ORF1 and actI-ORF2), an acyl carrier protein (ACP; actI-ORF3), and a malonylCoA:ACP transacylase (MAT) which is shared between polyketide and fatty acid synthesis (Carreras and Khosla 1998; Dreier et al. 1999). The resulting backbone is tailored into actinorhodin by the action of various enzymes, including ketoreductases, cyclases, and oxygenases, which are important for generating structural diversity (Rix et al. 2002). The cellular locations of polyketide tailoring enzymes have not been investigated in detail, but they are assumed to reside in the cytoplasm or to be bound to the inner part of the membrane, because polyketide biosynthesis occurs intracellularly. Therefore, it is not clear if the detection of some polyketide tailoring enzymes such as the ActVI-ORF3 dehydrase (Hesketh et al. 2002) and the ActIII ketoreductase (Xu et al. 2008) in the cell-wall fraction of S. coelicolor should be considered as an anomaly or not. In particular, ActIII (SCO5086) has received a special attention due to its uncertain cellular localization. This protein, which has 
one predicted transmembrane segment, was found in the cytoplasmic fraction in a proteomic analysis by Hesketh and Chater (2003). However, Xu and co-workers (2008) were not able to detect the actinorhodin ketoredutase ActIII in the cytoplasmic protein fraction using Western blotting analyses. In contrast, after careful analysis of proteins in subproteomes, these authors found ActIII to be associated with the cell wall. To clarify the localization of this protein in vivo, we have examined the spatial and temporal expression pattern of actIII using the green fluorescence protein

Table 1 Bacterial strains and plasmids used in this work

\begin{tabular}{|c|c|c|}
\hline Strains & Characteristics & Reference or source \\
\hline S. coelicolor M145 & Wild type & Kieser et al. (2000) \\
\hline E. coli $\mathrm{DH} 5 \alpha$ & $\mathrm{F}^{\prime} \Phi 80 d L a c Z \Delta \mathrm{M} 15$ & Hanahan (1983) \\
\hline E. coli $\mathrm{ET} 12567$ & (pUZ8002) dam dcm mutant, $\mathrm{Neo}^{\mathrm{r}}-\mathrm{Cm}^{\mathrm{r}}$ & MacNeil et al. (1992) \\
\hline \multicolumn{3}{|l|}{ Plasmids } \\
\hline pIJ8660 & Integrative promoter-probe vector, EGFP gene, $\mathrm{Am}^{\mathrm{r}}$ & Sun et al. (1999) \\
\hline pSCO5083-EGFP & $B g l \mathrm{II}-N d e \mathrm{I}$ SCO5083 fragment cloned into pIJ8660, $\mathrm{Am}^{\mathrm{r}}$ & This work \\
\hline pSCO5086-EGFP & $B g l \mathrm{II}-N d e \mathrm{I}$ SCO5086 fragment cloned into pIJ8660, $\mathrm{Am}^{\mathrm{r}}$ & This work \\
\hline \multicolumn{3}{|l|}{ Primers } \\
\hline FSB66 & 5'CTTCAGATCTCTCGCTTCGCGACACGTGCTCCTCATCGTATG & This work \\
\hline FSB67 & 5'GTCACCGCATATGGCCGCTCCCGGAGAAGCCCTCTTCCTCACGCGGCTTGGGCGGCAG & This work \\
\hline FSB68 & 5'CAGGAGATCTGACGAACATCGCGGCTCCTTCGGCCAGCACGAAG & This work \\
\hline FSB69 & 5'GTCACCGCATATGGCCGCTCCCGGAGTAGTTCCCCAGCCCGCCGCAGACGTTCAGCGC & This work \\
\hline
\end{tabular}

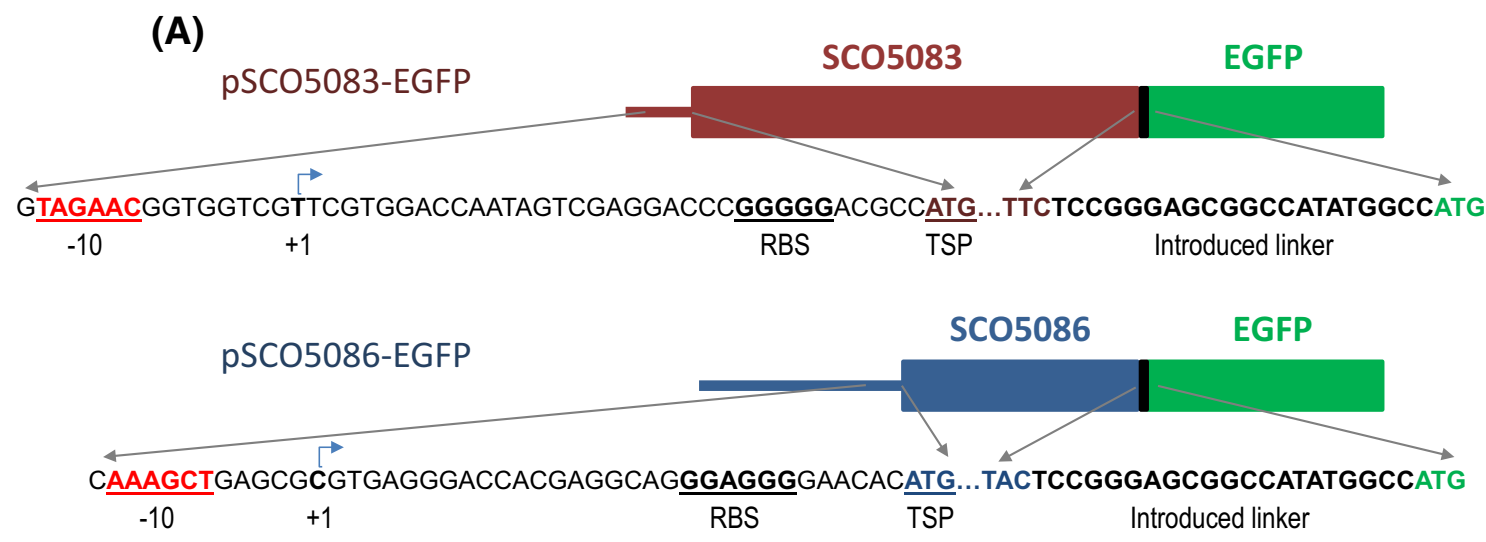

(B)
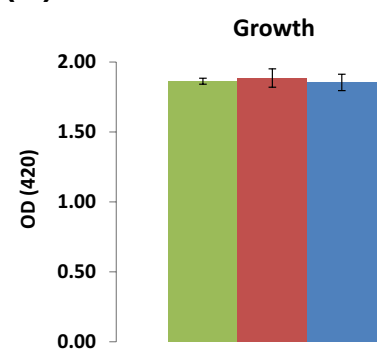

(C)

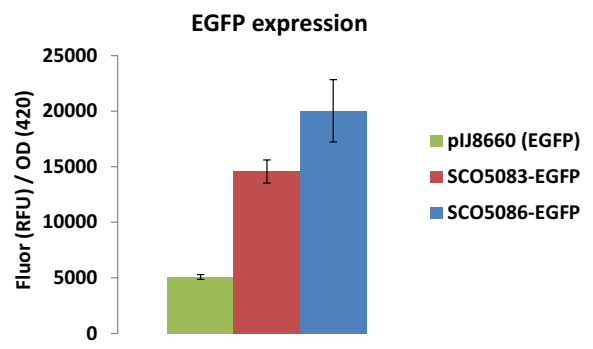

Fig. 1 a Scheme of the SCO5083-EGFP and SCO5086-EGFP fusion protein constructions. The black line between the labelled coding regions corresponds to the introduced amino acid linker composed of Ser, Gly, Ser, Gly, His, Met, and Ala. RBS: ribosomal binding site. TSP: translation start point $\mathbf{b}, \mathbf{c}$ Fluorescence reporter assay to quantify the expression of the SCO5083-EGFP and SCO5086-EGFP fusion proteins. Cells of the indicated strains were grown in TSA using black polystyrene sterile 96-well plates. Each well (containing $100 \mu \mathrm{L}$ of medium) was inoculated with $4 \mu \mathrm{L}$ of a stock dilution containing $10^{6}$ spores of $S$. coelicolor. Plates were then incubated at $30^{\circ} \mathrm{C}$ for $60 \mathrm{~h}$ before being assayed for growth (b) or EGFP expression (c). Vertical error bars correspond to the standard error of the mean of 24 replicate cultures 
and epifluorescence microscopy. We also report a study of actA (SCO5083), which codes for one of the three putative export pumps identified within the act cluster (Tahlan et al. 2007), as a control for a peripheral membrane protein.

\section{Materials and methods}

\section{Bacterial strains and plasmids}

The bacterial strains, plasmids, and primers used in this work are listed in Table 1. S. coelicolor strains were manipulated according to the standard procedures (Kieser et al. 2000). Escherichia coli DH5 $\alpha$ was the general cloning host. Cloning procedures were performed as described by Sambrook et al. (1989). The SCO5083 (actA) and SCO5086 (actIII) inserts were amplified by PCR using total DNA as template, as follows. The primers FSB66 and FSB67 amplified an $1894 \mathrm{bp}$ fragment encompassing the actA ORF (not including the stop codon) and the promoter region of the gene from +1 to +125 (positions from the ATG translation start triplet). Primers FSB68 and FSB69 were used to amplify an $1762 \mathrm{bp}$ fragment encompassing the actIII ORF (not including the stop codon) and the promoter region of the gene from positions +1 to +944 (positions from the translation start triplet). In both cases, the BglII (forward primer) and $N d e \mathrm{I}$ (reverse primer) cloning sites were introduced via the primer sequences. BglII-NdeI fragments were cloned into pIJ8660 (Sun et al. 1999) to obtain pSCO5083EGFP and pSCO5086-EGFP, respectively. The inserts of both plasmids were checked by sequencing.

\section{Growth conditions}

Streptomyces coelicolor solid cultures were performed in TSA (tryptone soya agar) using black polystyrene sterile 96-well plates. Each well (containing $100 \mu \mathrm{L}$ of medium) was added $4 \mu \mathrm{L}$ of a stock dilution containing $10^{6}$ spores of $S$. coelicolor. Plates were then incubated at $30^{\circ} \mathrm{C}$ during $60 \mathrm{~h}$. S. coelicolor liquid cultures were performed in defined MG-18.5 medium (Santos-Beneit et al. 2008). $100 \mathrm{ml}$ of MG-18.5 medium in $500 \mathrm{ml}$ baffled flasks were inoculated with $10^{6}$ spores per $\mathrm{ml}$ and incubated at $30^{\circ} \mathrm{C}$ in an orbital shaker for reproducible and dispersed growth.

\section{Growth and antibiotic production determinations}

Samples for growth and actinorhodin production determinations from the liquid cultures were taken after 26, 35 , 46,68 , and $90 \mathrm{~h}$ of incubation. Growth was determined by dry weight (culture samples of $2 \mathrm{ml}$ were washed twice with MilliQ water and dried for 4 days at $80^{\circ} \mathrm{C}$ before the weight measurements). Growth in solid media was determined by optical density $(420 \mathrm{~nm})$ using a BMG Fluostar Optima fluorometer. Actinorhodin quantification was performed, as described by Kieser et al. (2000).

\section{Fluorescence intensities determination}

The measurements were carried out at $30^{\circ} \mathrm{C}$ using a BMG Fluostar Optima fluorometer as follows: Fluorescence Top Reading mode; multiple reads per well; excitation wavelength $(485 \mathrm{~nm})$; Emission wavelength $(535 \mathrm{~nm})$.

\section{Fluorescence microscopy}

To visualise growing mycelia of $S$. coelicolor strains, cells were immobilized on microscope slides covered with a thin film of $1.2 \%$ agarose in water. Standard fluorescence microscopy was carried out using a Nikon Eclipse Ti microscope with a Nikon Plan Fluor $\times 100 / 1.30$ Oil objective. The images were acquired using MetaMorph (Molecular Devices) and further analysed using ImageJ (National Institutes of Health).

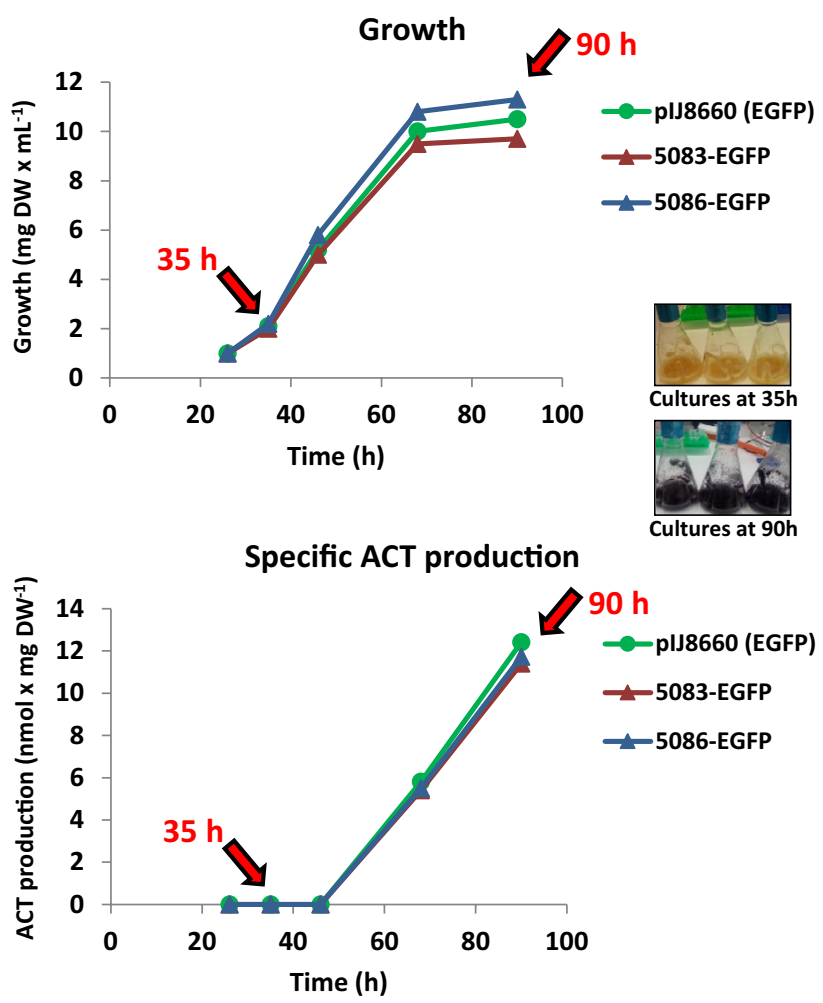

Fig. 2 Growth (upper panel) and actinorhodin (ACT) production (lower panel) of $S$. coelicolor M145 strains carrying pIJ8660, pSCO5083-EGFP or pSCO5086-EGFP vectors. Arrows indicate the times at which samples for phase contrast and epifluorescence microscopy were taken 


\section{Results and discussion}

\section{Constructs for actA and actIII-EGFP fusion proteins}

To obtain a construct expressing actA-EGFP and actIIIEGFP, we used the integrative plasmid pIJ8660 (Sun et al. 1999), which contains a promoterless enhanced version of the GFP coding gene (EGFP). The SCO5083 (actA) and SCO5086 (actIII) genes, including their own promoters (Parro et al. 1991; Caballero et al. 1991), were amplified by PCR from S. coelicolor M145 total DNA. BglII and NdeI digested DNA fragments were then introduced in pIJ8660 to obtain pSCO5083-EGFP and pSCO5086-EGFP final vectors. For both SCO5083 and SCO5086 amplifications,
(A) pIJ8660 (Phase)

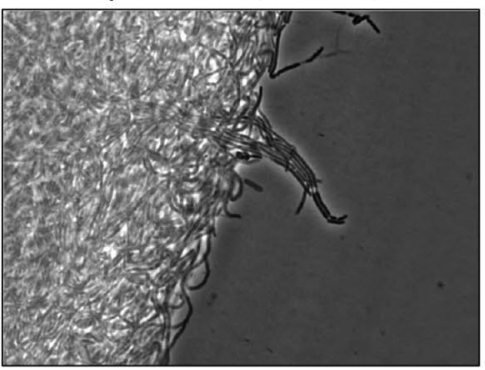

plJ8660 (Fluor)

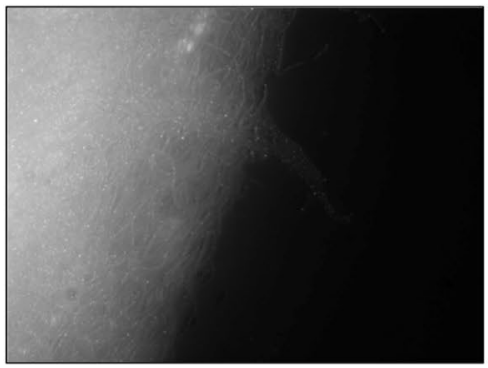

(B) plJ8660 (Phase)

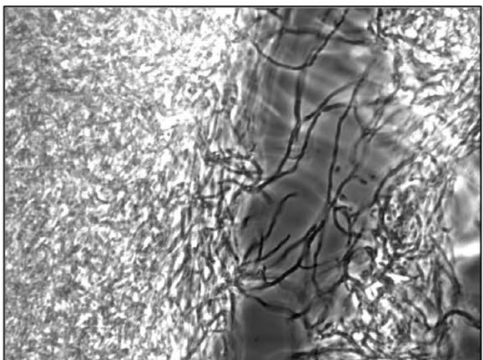

plJ8660 (Fluor)

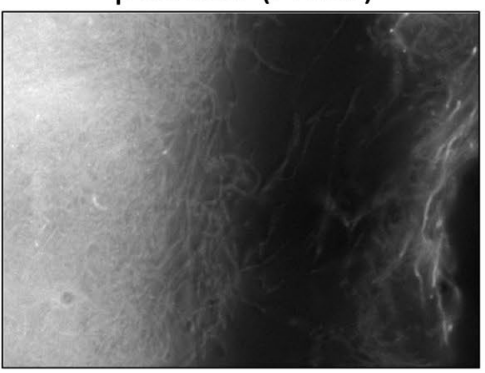

SCO5083-EGFP (Phase)
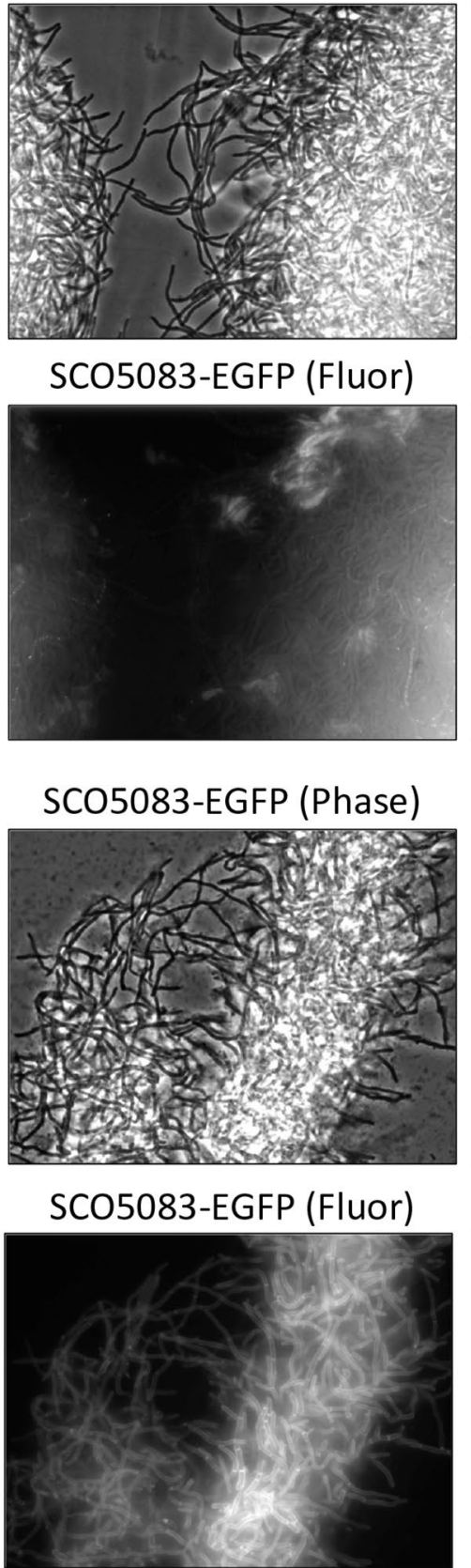

SCO5086-EGFP (Phase)

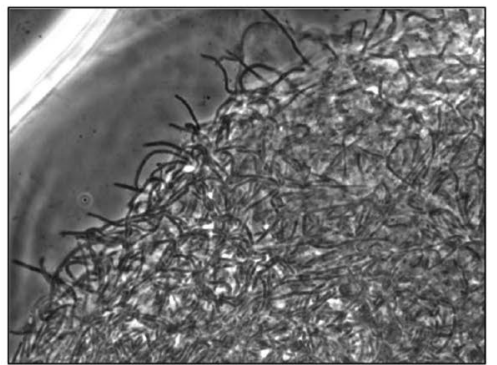

SCO5086-EGFP (Fluor)

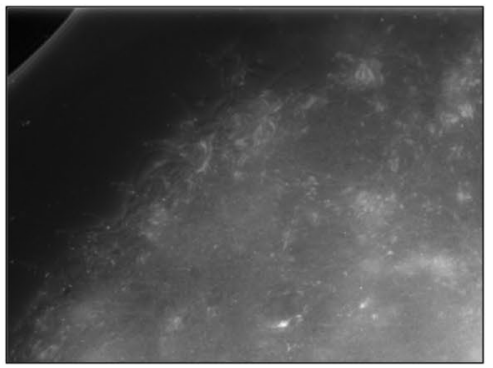

SCO5086-EGFP (Phase)

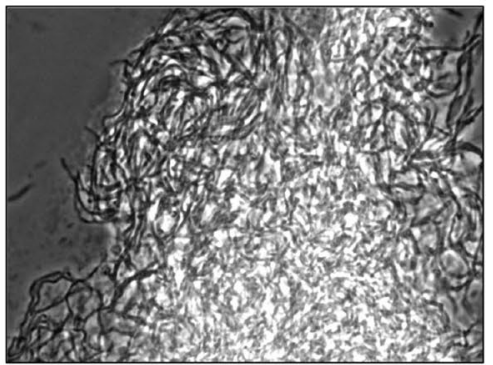

SCO5086-EGFP (Fluor)

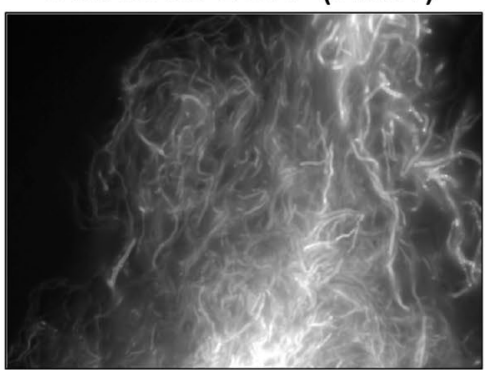

Fig. 3 Localization of SCO5083-EGFP and SCO5086-EGFP expressed from their native locus in S. coelicolor M145. Cultures were incubated in MG-18.5 medium at $30^{\circ} \mathrm{C}$ for $35 \mathrm{~h} \mathrm{(a)} \mathrm{or} 90 \mathrm{~h} \mathrm{(b).}$ In each part, the upper panels correspond to phase contrast images and the lower panels to fluorescence images. Note that the exposure time for the fluorescence images in samples at $35 \mathrm{~h}$ was three times longer than in samples at $90 \mathrm{~h}$ (3000 and $1000 \mathrm{~ms}$, respectively) 
the $B g l I I$ restriction sequence was added to the 5 ' region of the direct primer. In both cases, the reverse primers contained the last $33 \mathrm{bp}$ of the ORF (not including the end codon) and a $5^{\prime}$ tail with the NdeI site. For both constructions, the tail contained the same sequence coding for a specific 6 aa-peptide. In this manner, the cloning of the respective fragments to the BglII/NdeI site of the vector left a Ser, Gly, Ser, Gly, His, Met, and Ala amino acid linker between the cloned gene and EFGP (note that there is an additional triplet for Ala in the pIJ8660 vector after the ATG of the NdeI site and before the start codon triplet of the EGFP; see Fig. 1a). A transcriptional terminator upstream of the cloning site avoids polar effects on the cloned genes, so that expression of the fusion proteins in pSCO5083-EGFP and pSCO5086-EGFP solely depends on the natural promoters of SCO5083 and SCO5086, respectively (see "Material and methods").

\section{SCO5083-EGFP and SCO5086-EGFP fusions are expressed in $S$. coelicolor TSA cultures}

To check if the fusion proteins were indeed expressed, we made fluorescent reporter analyses in black polystyrene microtiter plates using a BMG Fluostar Optima fluorometer (see "Material and methods"). To carry out the assay, $S$. coelicolor spores $\left(10^{6}\right.$ in all cases) containing either pIJ8660, pSCO5083-EGFP or pSCO5086-EGFP vectors were grown for $60 \mathrm{~h}$ in TSA medium (supplemented with $50 \mu \mathrm{g} / \mathrm{ml}$ apramycin to maintain selective pressure on the plasmids), until actinorhodin production was evident. The growth yields at this timepoint were estimated by measuring $\mathrm{OD}_{420}$ in each well; mean values of 24 replicates for each of the strains used are shown in Fig. 1b. Protein expression was given in fluorescence specific values which were calculated as a ratio of relative fluorescence units and OD (Fig. 1c). Fluorescence values were 3-4 times higher in the strains carrying the fusion proteins than in the strain carrying the promoterless EGFP protein, indicating that both SCO5083-EGFP and SCO5086-EGFP fusions were expressed.

\section{Localization of SCO5083 (ActA) and SCO5086 (ActIII) proteins}

As mentioned in the "Introduction", previous descriptions of the cellular localization of ActIII were conflicting. However, the membrane localization of ActA was expected to be unambiguously membrane associated as it is predicted to possess 13 transmembrane segments (Hesketh and Chater 2003). This protein was, therefore, expected to provide a membrane localization control for our analyses. The SCO5083-EGFP fluorescence signal should also demonstrate whether the fusion protein is stable and if the fusion protein linker is flexible enough to permit folding and activation of the fluorescent moiety.

Streptomyces coelicolor cells containing pIJ8660, pSCO5083-EGFP, and pSCO5086-EGFP were grown in MG-18.5 medium (Santos-Beneit et al. 2008) in which undecylprodigiosin production (the other pigmented antibiotic produced by $S$. coelicolor) is almost suppressed, but not actinorhodin (Santos-Beneit et al. 2009). Growth and actinorhodin production were monitored during the time course of the experiment to identify samples corresponding to the actinorhodin production phase (see Fig. 2). Samples were selected at $35 \mathrm{~h}$ (production off) and $90 \mathrm{~h}$ (production on). No significant differences in fluorescence were
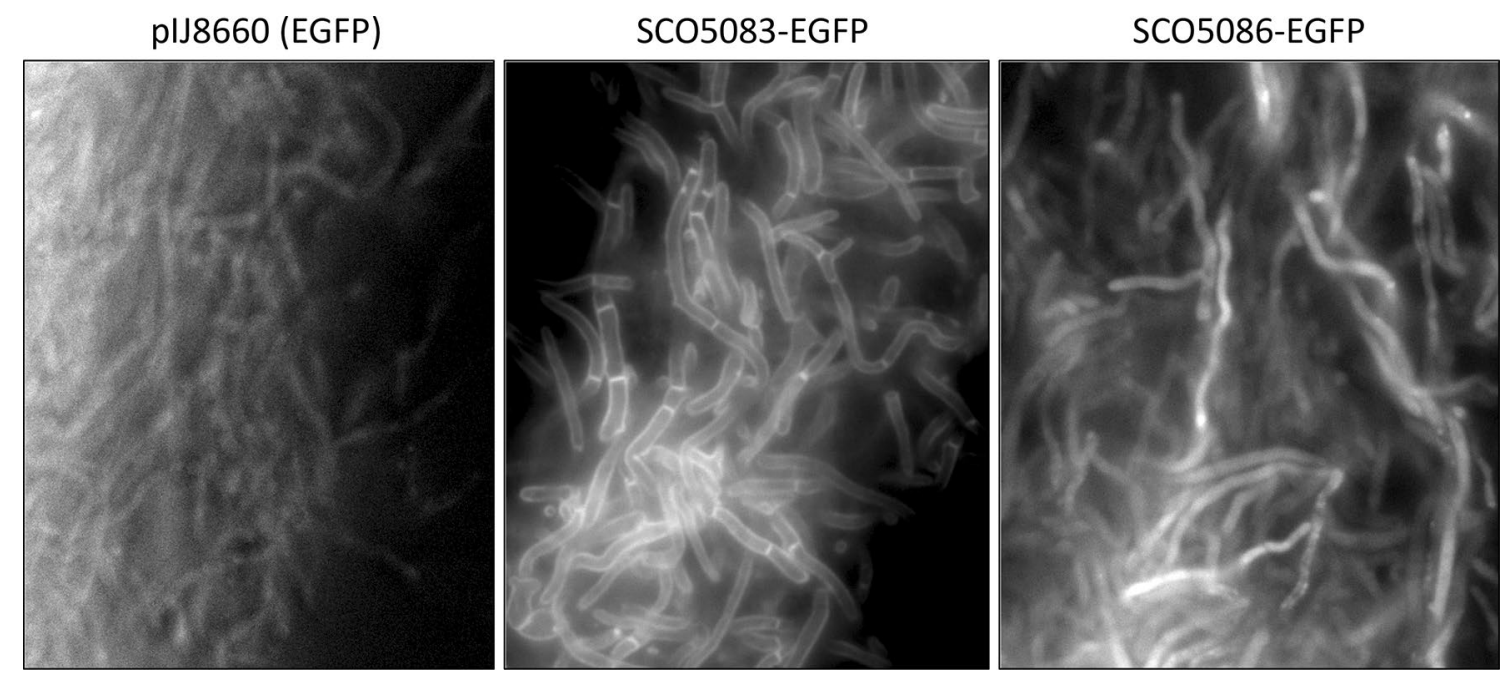

Fig. 4 Detail of EGFP expression in S. coelicolor M145 cells, containing pIJ8660 (control) pSCO5083-EGFP and pSCO5086-EGFP vectors, cultures in MG-18.5 medium for $90 \mathrm{~h}$. All of the fluorescence images were obtained with an exposure time of $1000 \mathrm{~ms}$ 
observed between the 3 strains in the $35 \mathrm{~h}$ samples (see Fig. 3a). However, fluorescence was much higher in the cells containing either pSCO5083-EGFP or pSCO5086EGFP when the samples at $90 \mathrm{~h}$ were analysed (even using three times lower exposure times than in the $35 \mathrm{~h}$ samples; see Fig. 3b). The negative control ( $S$. coelicolor harbouring the pIJ8660 vector), which grew and produced actinorhodin similarly to the two other strains (see Fig. 2), failed to show any increase in the fluorescence signal. This result was in agreement with the temporal expression patterns expected for ActIII and ActA; i.e., the proteins should only be expressed during the actinorhodin production phase.

In relation to the spatial expression patterns, the SCO5083EGFP fusion protein localized specifically to the membrane of the $S$. coelicolor cells, including not only the periphery of the cells but also the vegetative cross-walls (see Fig. 4). However, the localization of the pSCO5086-EGFP fusion protein was completely different to that of pSCO5083-EGFP; the fluorescence was observed within the cell body rather than only in the periphery. This fluorescence pattern for pSCO5086-EGFP strongly suggested that ActIII is a cytosolic protein, with limited if any association with the membrane or cell wall. We cannot formally exclude the possibility that the native (i.e., non-EGFP) SCO5086 protein could have a different localization pattern but given that the pool of the wild type and fusion proteins supports the same actinorhodin production yields than the wild-type protein, it seems that the cytosolically localized protein is functional.

Acknowledgements This work was funded by a Wellcome Trust Senior Investigator Award (WT098374AIA) to JE.

Open Access This article is distributed under the terms of the Creative Commons Attribution 4.0 International License (http://creativecommons.org/licenses/by/4.0/), which permits unrestricted use, distribution, and reproduction in any medium, provided you give appropriate credit to the original author(s) and the source, provide a link to the Creative Commons license, and indicate if changes were made.

\section{References}

Caballero JL, Malpartida F, Hopwood DA (1991) Transcriptional organization and regulation of an antibiotic export complex in the producing Streptomyces culture. Mol Gen Genet 228:372-380

Carreras CW, Khosla C (1998) Purification and in vitro reconstitution of the essential protein components of an aromatic polyketide synthase. BioChemistry 37:2084-2088

Dreier J, Shah AN, Khosla C (1999) Kinetic analysis of the actinorhodin aromatic polyketide synthase. J Biol Chem 274:25108-25112

Fernandez-Moreno MA, Martinez E, Caballero JL, Ichinose K, Hopwood DA, Malpartida F (1994) DNA sequence and functions of the actVI region of the actinorhodin biosynthetic gene cluster of Streptomyces coelicolor A3(2). J Biol Chem 269:24854-24863

Hanahan D (1983) Studies on transformation of Escherichia coli with plasmids. J Mol Biol 166:557-580

Hesketh A, Chater KF (2003) Evidence from proteomics that some of the enzymes of actinorhodin biosynthesis have more than one form and may occupy distinctive cellular locations. J Ind Microbiol Biotechnol 30:523-529

Hesketh AR, Chandra G, Shaw AD, Rowland JJ, Kell DB, Bibb MJ, Chater KF (2002) Primary and secondary metabolism, and posttranslational protein modifications, as portrayed by proteomic analysis of Streptomyces coelicolor. Mol Microbiol 46:917-932

Hopwood DA (1997) Genetic contributions to understanding polyketide synthases. Chem Rev 97:2465-2498

Keatinge-Clay AT, Maltby DA, Medzihradszky KF, Khosla C, Stroud RM (2004) An antibiotic factory caught in action. Nat Struct Mol Biol 11:888-893

Kieser T, Bibb MJ, Buttner MJ, Chater KF, Hopwood DA (2000) Practical Streptomyces genetics. The John Innes Foundation, Norwich

MacNeil DJ, Gewain KM, Ruby CL, Dezeny G, Gibbons PH, MacNeil T (1992) Analysis of Streptomyces avermitilis genes required for avermectin biosynthesis utilizing a novel integration vector. Gene 111:61-68

Malpartida F, Hopwood DA (1986) Physical and genetic characterisation of the gene cluster for the antibiotic actinorhodin in Streptomyces coelicolor A3(2). Mol Gen Genet 205:66-73

Parro V, Hopwood DA, Malpartida F, Mellado RP (1991) Transcription of genes involved in the earliest steps of actinorhodin biosynthesis in Streptomyces coelicolor. Nucl Acids Res 19:2623-2627

Rawlings BJ (1999) Biosynthesis of polyketides (other than actinomycete macrolides). Nat Prod Rep 16:425-484

Rix U, Fischer C, Remsing LL, Rohr J (2002) Modification of postPKS tailoring steps through combinatorial biosynthesis. Nat Prod Rep 19:542-580

Sambrook J, Fritsch EF, Maniatis T (1989) Molecular cloning: a laboratory manual, 2nd edn. Cold Spring Harbor Laboratory, Cold Spring Harbor, NY

Santos-Beneit F, Rodríguez-García A, Franco-Domínguez E, Martín JF (2008) Phosphate-dependent regulation of the low- and highaffinity transport systems in the model actinomycete Streptomyces coelicolor. Microbiology 154:2356-2370

Santos-Beneit F, Rodríguez-García A, Sola-Landa A, Martín JF (2009) Cross-talk between two global regulators in Streptomyces: PhoP and AfsR interact in the control of afsS, pst $S$ and phoRP transcription. Mol Microbiol 72:53-68

Sun J, Kelemen GH, Fernández-Abalos JM, Bibb MJ (1999) Green fluorescent protein as a reporter for spatial and temporal gene expression in Streptomyces coelicolor A3(2). Microbiology 145:2221-2227

Tahlan K, Ahn SK, Sing A, Bodnaruk TD, Willems AR, Davidson AR, Nodwell JR (2007) Initiation of actinorhodin export in Streptomyces coelicolor. Mol Microbiol 63:951-961

Xu XP, Wang ZJ, Fan KQ, Wang SL, Jia CJ, Han H, Ramalingam E, Yang KQ (2008) Localization of the ActIII actinorhodin polyketide ketoreductase to the cell wall. FEMS Microbiol Lett 287:15-21 